\title{
Contactless Processing of Metallic Materials by Stabilized Electromagnetic Levitation
}

\author{
Pascale Gillon* \\ EPM Laboratory CNRS, 25 Avenue des Martyrs BP166, 38042 Grenoble Cedex France
}

\begin{abstract}
The paper presents a new containerless technique for metallic materials processing based on the simultaneous application of a high frequency electromagnetic field and an inhomogeneous DC magnetic field of strong intensity. It is shown how the superposition of the DC magnetic field strongly enhances the stability of the electromagnetic levitation. Levitation experiments on massive paramagnetic samples involving the combined magnetic and electromagnetic fields are described and analyzed. Conditions to solidify pure $\mathrm{Ti}$ and $\mathrm{Ti}-\mathrm{Al}$ alloys samples of $45 \mathrm{~g}$ without contact are presented.
\end{abstract}

(Received February 25, 2000; Accepted June 2, 2000)

Keywords: containerless processing, magnetic levitation, electromagnetic levitation, stability, undercooling

\section{Introduction}

Materials processing without contact with a container is of great interest and has been used for the fundamental study of melts, such as their thermophysical properties and their solidification behaviour. ${ }^{1)}$ Avoiding the introduction of impurities from walls, containerless techniques interest especially materials of high melting point, strongly reactive or requiring a high purity level. Because freezing process takes place in the absence of a container, the generally dominant heterogeneous nucleation on walls is completely eliminated. Furthermore, if experiments are performed in conditions of extreme purity, heterogeneous nucleation on free surfaces can be greatly reduced. In this way, undercooled melts below their equilibrium melting temperature can be obtained. Undercooled melts are in a non-equilibrium state in which the solidification is delayed. In those melts, the nucleation step prior to solidification is of particular interest as a decisive process in selecting the phase, stable or metastable, to be solidified. Moreover, containerless solidification has been proved to be benefit on crystal quality due to the reduction of mechanical constraints induced by the walls on the growing crystal.

Apart from microgravity environment, contactless processing can be realized either by the free fall of small droplets in drop tubes or by levitation. In the former case, the size of the droplets is limited because of the short experimental time during free fall. Levitation, obtained by compensating the gravitational force acting on the sample, offers the possibility of undercooling more massive samples accessible for direct observation and external stimulation. The most used method to suspend freely a metallic sample is the electromagnetic levitation (EML) technique. However, because EML systems suffer from a number of stability problems, ${ }^{2)}$ its range of applications is limited.

In the present paper, we describe how the superposition of an inhomogeneous DC magnetic field of strong intensity is able to stabilize electromagnetic levitation leading to a new

*E-mail: gillon@labs.polycnrs-gre.fr containerless process. To do this, we first detailed the electromagnetic levitation principles and the effects of a simultaneous DC magnetic field application. Stabilized levitation experiments of metallic melts are then presented and analyzed before describing the achievement of contactless solidification of massive metallic samples.

\section{Electromagnetic Levitation}

\subsection{Principle}

A typical EML device is sketched on Fig. 1. A highfrequency (typically $1-8 \times 10^{5} \mathrm{~Hz}$ ) current is passing through conically wound coils, generating an electromagnetic field. When a metallic sample is placed inside the coils, eddy currents will be induced in the sample. Interaction of these currents with the applied electromagnetic field produces a Lorentz force having two mechanical effects: a lifting effect able to support the metal against gravity and a turbulent fluid flow in the molten metal. At the same time, the induced currents produce heat following Joule's law which raises the metal temperature and may melt or even superheat the sample. Figure 2 presents the EML device used in the present experimental study. It consists of a water cooled sectorized metallic crucible of $32 \mathrm{~mm}$ inner diameter inserted inside a 4 turn cylindrical inductor; the semi-hemispherical shape of the crucible being responsible for the concentration of magnetic

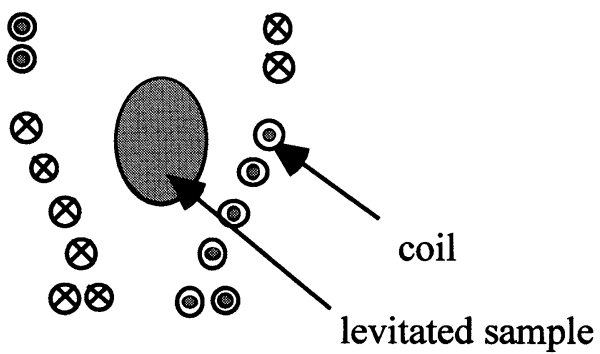

Fig. 1 Typical electromagnetic levitation (EML) system showing a conical coil arrangement: the lower coil provides the levitation force; the upper coil is a stabilizator against vertical displacements. 


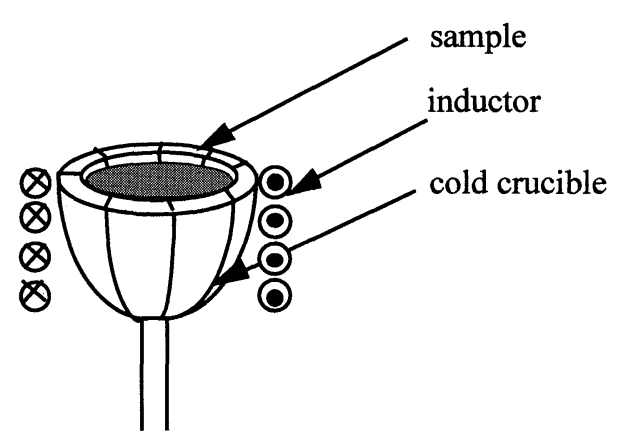

Fig. 2 Cold crucible levitation device of semi-hemispherical shape.

flux lines at the bottom of the device. In EML systems, the intensity of the electromagnetic field governs both the temperature of the levitated samples and the lifting force. To the levitation field necessary to compensate the gravitational force corresponds a power absorption within the levitated sample leading to the heating effect. Hence, only a limited range of temperature is achievable. Cooling of the sample has to overcome the heating effect and is generally obtained by convective cooling gases.

Despite obvious advantages revealed in the numerous studies using this levitation technique, practical applications in materials processing are limited mainly because of stability problems. Firstly, in symmetry axis configurations such as the one on Fig. 1, the magnetic field experiences stagnation points where it vanishes on the metal surface at the highest and lowest points. At the lowest point, the lifting force being null, the surface tension is solely responsible for supporting the column of fluid in this region. This is generally the crucial factor in limiting the mass of fluid metal that can be levitated. The molten metal in levitation is also subjected to instabilities due to the intense internal agitation which could lead to rapid vibrations or rotations of the sample and cause lateral contacts with the coils or the walls of the system.

\subsection{Superimposition of a DC magnetic field}

The superimposition of a strong D.C. field to an EML system will result in three stabilization effects leading to a large enhancement of its application range. The first one is that high static magnetic fields are able to levitate materials of weak magnetic properties. ${ }^{3,4)}$ The principle is the following: when a specimen of magnetic susceptibility $\chi$ is set in a magnetic field of induction $\boldsymbol{B}$ possessing a spatial gradient, a magnetic force develops which tends to drive it to regions of smaller energy. This magnetic force per unit mass is given by:

$$
\boldsymbol{F}_{\mathrm{m}}=\left(\chi / 2 \mu_{0}\right) \operatorname{grad}\left(\boldsymbol{B}^{2}\right)
$$

In S.I. units, $F_{\mathrm{m}}$ is in N/kg, $\operatorname{grad}\left(\boldsymbol{B}^{2}\right)$ in $\mathrm{T}^{2} / \mathrm{m}$ and $\chi$ in $\mathrm{m}^{3} / \mathrm{kg}$. The value of $\mu_{0}$ is $4 \pi \times 10^{-7}$. In the case of axisymmetric coils delivering a vertical magnetic field $\boldsymbol{B}_{z}$ on the axis, the vertical force is:

$$
\boldsymbol{F} \boldsymbol{m}_{\mathrm{z}}=\left(\chi / \mu_{0}\right) \boldsymbol{B}_{\mathrm{z}} \mathrm{d} \boldsymbol{B}_{\mathrm{z}} / \mathrm{d} z
$$

Equation (2) shows that the magnetic force direction depends on the sign of both the field gradient and the magnetic susceptibility $\chi$ of the specimen. An upward vertical force is obtained below the center of the coil for paramagnetic sub- stances $\left(\mathrm{d} \boldsymbol{B}_{\mathrm{z}} / \mathrm{d} z>0\right.$ and $\left.\chi>0\right)$ and above the center of the coil for diamagnetic ones $\left(\mathrm{d} \boldsymbol{B}_{\mathrm{z}} / \mathrm{d} z<0\right.$ and $\left.\chi<0\right)$. When the magnetic force compensates for gravity, levitation can occur. Considering radial stability off the axis, if there exists stable areas for levitation of diamagnetic specimen, it has been demonstrated ${ }^{5,6)}$ that magnetic levitation of paramagnetic samples is not stable due to centrifugal forces which tend to drive the levitated sample towards external walls. By superimposing an inhomogeneous D.C. magnetic field to an electromagnetically levitated metallic sample, either dia or paramagnetic, it is possible to add a vertical body force directed upwards. The magnetic force, being volumic, can compensate for gravity at the bottom point of the sample enhancing greatly the quantity which could be levitated.

Classical magnetohydrodynamic effect of damping of motions also play an important role in the dynamic stabilization of EML. Both the global motions of the sample, solid or liquid, and the internal fluid flow induced by the high frequency magnetic field inside the molten metal are braked by the strong D.C. magnetic field. The braking mechanism lies in the existence of a Lorentz force $\boldsymbol{j} \times \boldsymbol{B}$ where $\boldsymbol{j}$ is the eddycurrent density driven by the electromotive field $\boldsymbol{u} \times \boldsymbol{B}(\boldsymbol{u}$ being the fluid velocity). From this definition of the braking Lorentz force it has to be noted that the braking effect is anisotropic, motions parallel to the magnetic field are not affected.

The third aspect introduced by the application of the D.C. magnetic field is the partial decoupling of the lift and thermal effects. Because the D.C. magnetic field provides another source of lifting force, it becomes possible to monitor the sample temperature with the intensity of the electromagnetic field and to compensate the variation of the electromagnetic lifting force by an equivalent variation of the magnetic levitation force.

Finally, the simultaneous application of a D.C. and an A.C. magnetic field produced the conditions for radial stability of the metallic sample in levitation. The centrifugal forces off axis due to the D.C. field are counterbalanced by the centripetal forces generated electromagnetically on the sample.

\section{Experimental}

\subsection{Experimental apparatus and procedure}

A schematic of the apparatus is presented on Fig. 3. It consists of the cold crucible induction furnace described previously within a vacuum chamber allowing to work under a purified gas atmosphere such as Ar or He. The coil is connected to an aperiodic power supply of $30 \mathrm{~kW}$ delivering an alternating current of $90-300 \mathrm{kHz}$ frequency range. The EML device is inserted inside the $120 \mathrm{~mm}$ diameter bore of a Oxford Instruments superconducting coil able to generate up to $8 \mathrm{~T}$. Profiles of typical field $\boldsymbol{B}$ and field $x$ field gradient product $\boldsymbol{B} \times \mathrm{d} \boldsymbol{B} / \mathrm{d} z$ are presented on Fig. 4 showing the vertical locations of the two maximum force values. The EML system is supported on a vertical translation stage in order to position the sample in one of the two D.C. magnetic field gradient areas. A video camera records from the top the behaviour of the sample and its surface temperature is supervised by an infrared two color pyrometer with a relative accuracy of $5 \mathrm{~K}$. 


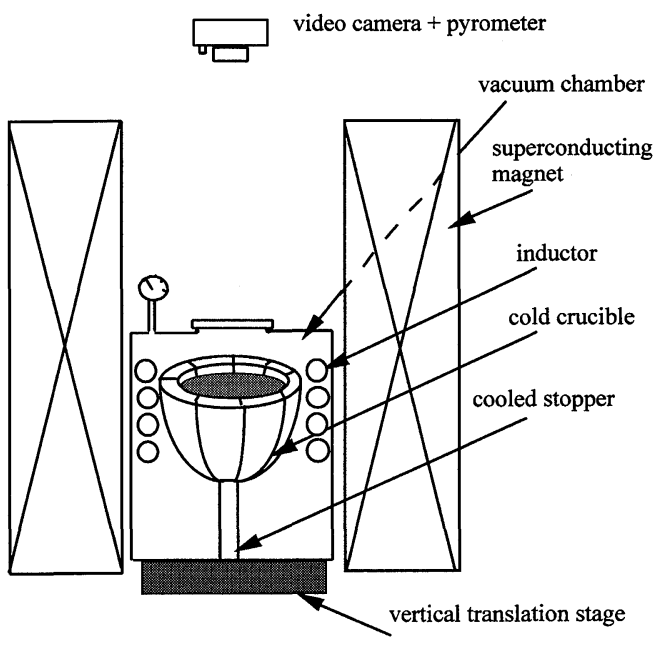

Fig. 3 Schematic of the experimental apparatus associating D.C. and high frequency magnetic fields.

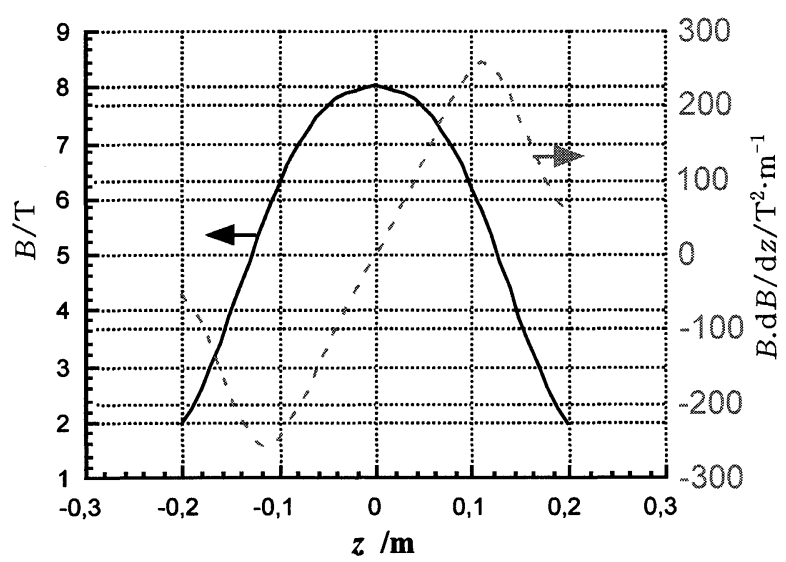

Fig. 4 Vertical distribution of the field and field $x$ field gradient product on the axis of the superconducting coil for $8 \mathrm{~T}$ delivered at the center $z=0$. The vertical force is upwards on diamagnetic materials for $z>0$ and on paramagnetic materials for $z<0$.

Levitation experiments were performed on different metaland alloy-samples whose size was defined by the capacity of the cold crucible i.e. about $10^{-5} \mathrm{~m}^{3}$. The sample was placed inside of the cold crucible and the chamber is evacuated prior to the injection of a purified argon gas. Before the experiment begins, the EML system has been set at the vertical position in the superconducting coil corresponding to the application of a magnetic levitation force on the sample. The metal is first heated by induction without the D.C. magnetic field. Once melted, the D.C. field was progressively increased until the sample raises inside the crucible. Levitation was detected by the observations of free movements of the metal with respect to the fixed crucible associated to a raise in the surface temperature as soon as the sample quits the cold crucible and brakes thermal contacts.

The contactless solidification procedure has to take into account the thermal behaviour of the sample. The cooling is obtained by decreasing the high frequency magnetic field with two consequent effects: one is the associated decrease of the electromagnetic lifting force; the other is the variation of the magnetic force with the sample temperature through the variation of the magnetic susceptibility. Levitation is maintained along the cooling by a continuous variation of the D.C. magnetic field intensity in order to provide a constant total lifting force.

\subsection{Levitation experiments}

Levitation experiments have been performed on samples of pure metals with different magnetic and electrical properties: $\mathrm{Cu}, \mathrm{Co}, \mathrm{Fe}, \mathrm{Ni}$ and $\mathrm{Ti}$. Copper is diamagnetic with a very low susceptibility which varies weakly with temperature. Cobalt, iron, nickel show a paramagnetic behaviour above the Curie point whose temperature dependence follows the Curie's law $\left(\chi \propto T^{-1}\right)$. Titanium is also paramagnetic but its susceptibility increases with temperature.

During the first step of the experiment, only the alternating magnetic field is applied. Once the sample is melted, it presents a spheroid shape in contact with the bottom of the crucible and intense internal agitation and free surface deformations due to the electromagnetic stirring forces. As soon as the static field is applied even at very low magnetic field intensity, a stabilization of the sample is observed. The internal motions are progressively damped by Lorentz forces and the sample presents a stable shape. Then, at increasing value of $\boldsymbol{B}$, the upward magnetic force pull the sample which elongates along the $z$-axis to take the shape of an egg. A further increase of the amplitude of the magnetic force induces levitation of the molten metal characterized by free movements of the sample with respect to the crucible.

In the range of parameters of the experiment, we have not been able to produce levitation of the $\mathrm{Cu}$ sample. For $\mathrm{Co}$, $\mathrm{Fe}$ and Ti samples stable levitation has been obtained both in solid and melted state with the combined A.C. and D.C. fields. Levitation of the $\mathrm{Ni}$ samples has been more delicate to produce due to the presence of deformations of the liquid droplet causing contacts with the crucible even when the D.C. field is applied.

Conditions of experiments for the paramagnetic samples are shown in Table 1. Samples of $\mathrm{Co}, \mathrm{Ni}$ and Ti 1 are solid, whereas $\mathrm{Fe}$ and Ti 2 are liquid at a temperature just above their melting point. At the reported conditions, $\mathrm{Fe}, \mathrm{Ni}$ and Ti 1 were in stable levitation; Co and Ti 2 have been expulsed from the crucible by an upward force of too strong intensity. Calculations of the electromagnetic and magnetic components of the levitation force based on the experimental data are given in Table 2 along with the sum of the two components. The magnetic force intensity has been deduced from the field gradient value and the magnetic susceptibility of the sample estimated at the temperature measured experimentally. The electromagnetic component was calculated, considering a spherical sample, using the analytical model proposed by Fromm and Jehn ${ }^{7)}$ adapted to the present cold crucible configuration as detailed in ${ }^{8)}$ and validated on ambient temperature measurements. Calculated results are consistent with the experimental observations corresponding to a total force of $9.81 \mathrm{~N} / \mathrm{kg}$ for the levitation cases. They show that the magnetic force is the dominant levitation force particularly for the high magnetic susceptibility metals. The systematic under estimation of the calculated values is attributed 
Table 1 Levitation conditions for different metallic samples; $\chi_{\mathrm{T}}$ is the massic susceptibility at temperature $T, U$ the power supply output tension, $B \mathrm{~d} B / \mathrm{d} z$ is the value corresponding to the chosen vertical position in the coil.

\begin{tabular}{ccrccc}
\hline & $\begin{array}{c}\text { mass } \\
(\mathrm{kg})\end{array}$ & \multicolumn{1}{c}{$\begin{array}{c}{ }^{\circ} \mathrm{C} \\
\text { Cobalt }\end{array}$} & $\begin{array}{c}\chi_{\mathrm{T}} \\
\left(\mathrm{m}^{3} / \mathrm{kg}\right)\end{array}$ & $\begin{array}{c}U \\
(\mathrm{kV})\end{array}$ & $\begin{array}{c}B \mathrm{~d} B / \mathrm{d} z \\
\left(\mathrm{~T}^{2} / \mathrm{m}\right)\end{array}$ \\
\hline Iron & $70.67 \times 10^{-3}$ & 1420 & $83 \times 10^{-8}$ & 2.5 & 11.3 \\
Nickel & $72.64 \times 10^{-3}$ & 880 & $12.8 \times 10^{-8}$ & 2.5 & 84.06 \\
Titanium 1 & $43.8 \times 10^{-3}$ & 1630 & $5.03 \times 10^{-8}$ & 3.3 & 180 \\
Titanium 2 & $43.8 \times 10^{-3}$ & 1670 & $5.09 \times 10^{-8}$ & 3.5 & 190 \\
\hline
\end{tabular}

Table 2 Electromagnetic and magnetic levitation components deduced from the experimental data of Table 1.

\begin{tabular}{cccc}
\hline & $\begin{array}{c}F \\
(\mathrm{~N} / \mathrm{kg})\end{array}$ & $\begin{array}{c}F m \\
(\mathrm{~N} / \mathrm{kg})\end{array}$ & $\begin{array}{c}\text { Total levitation force } \\
(\mathrm{N} / \mathrm{kg})\end{array}$ \\
\hline Cobalt & .59 & 9.04 & 9.63 \\
Iron & .86 & 8.15 & 9.01 \\
Nickel & .71 & 8.57 & 9.28 \\
Titanium 1 & 1.9 & 7.2 & 9.1 \\
Titanium 2 & 2.14 & 7.7 & 9.84 \\
\hline
\end{tabular}

to the non-spherical shape of the real levitated drop and to the variation of the sample position and temperature inside the two coils. Calculations also show that the association of the two types of magnetic field does not influenced each levitation phenomena; the resulting levitation force being the sum of the applied two forces.

\subsection{Contactless solidification experiments}

Contactless solidification of pure Ti and Ti-Al 50 at $\%$ samples was obtained by using the protocol described above. The samples were cooled by a continuous decrease of the A.C. power while levitation was maintained by a simultaneous increase in the D.C. intensity. The first step of the solidification process was detected both by visual observations and surface temperature measurements. Solidification initiates at the top and the bottom metal surface where less inductive power is injected and thermal losses by radiation are higher. The freezing process was at the sample equator leaving on the pure metal surface a line where the two solidification fronts have met. In the case of $\mathrm{Ti}-\mathrm{Al}$ alloys, solidification shrinkage induced holes and internal porosities were always observed in this specific equator area. The thermal measurements have shown a sudden increase of surface temperature corresponding to the release of the latent heat of solidification followed by a much slower decrease of the sample temperature due to the small thermal transport from the metal surface to the atmosphere in the contactless conditions. A completely smooth surface of the egg-shaped samples attests of a non contact solidification as shown on Fig. 5. However, due to heterogeneous nucleation on the free surface, high undercooling has not been observed in our experiments. The microstructure is characterized by optical metallography. The sample was sectionned along the magnetic field direction, polished and etched with a nitric-fluoric acid. Figure 6 shows a dendritic structure characterisitic of a quiescient melt.

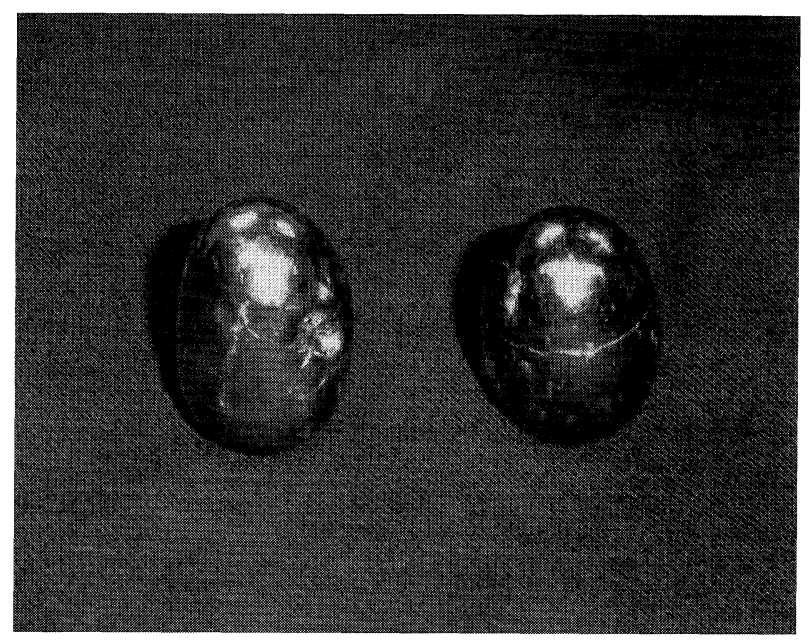

Fig. 5 Photograph of two samples produced by contactless solidification: right pure Ti and left $\mathrm{Ti}-\mathrm{Al}$ alloy.

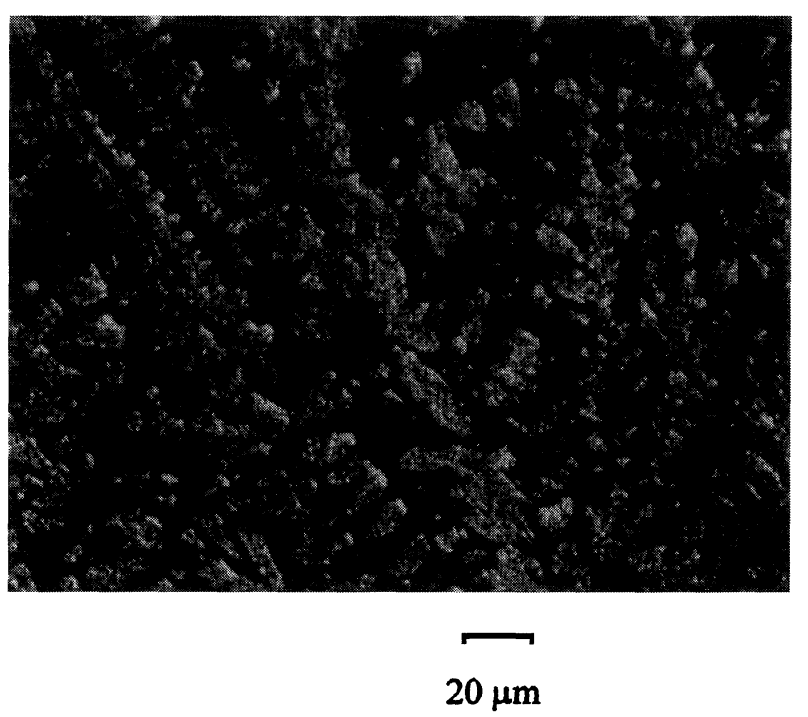

Fig. 6 Microstructure of the TiAl 50 at $\%$.

\section{Conclusions}

As expected, the superimposition of a strong inhomogeneous magnetic field to a EML system has enhanced the domain of stability of levitation of metallic materials, allowing to treat bigger quantities of materials and offering the possibility of contactless solidification processing. Contactless solidifcation has been obtained by a control of the sample temperature through the inductive input power while keeping constant the resultant levitation effect. Using this technique, high solidification rates cannot be obtained. Larger sample sizes induce larger heat released during solidification which cannot be rapidly evacuated from the sample due to the small thermal transport in non contact conditions. Influence of the stabilization of the melt flow on the resulting solidification microstructure is under study. 


\section{REFERENCES}

1) D. M. Herlach, R. F. Cochrane, I. Egry, H. J. Fecht and A. L. Greer: Int. Matls Rev., 38 (1993), 273-347.

2) A. D. Sneyd and H. K. Moffatt: J. Fluid Mech., 117 (1982), 45-70.

3) E. Beaugnon and R. Tournier: J. Phys. III France, 1 (1991), 1423-1428.

4) E. Beaugnon, D. Bourgault, D. Braithwaite, P. de Rango, R. Perrier de la Bathie, A. Sulpice and R. Tournier: J. Phys. I France, 3 (1993), 399-421.

5) T. B. Jones: J. Appl. Phys., 50 (1979), 5057-5058.

6) L. M. Holmes: J. Appl. Phys., 49 (1978), 3102-3109.

7) E. Fromm and H. Jehn: Brit. J. Appl. Phys., 16 (1965), 653-663.

8) P. Gillon: Proceeding EPM'97 International Conference Paris CFE editors. vol. 1, (1997), 137-142. 